\title{
Thoracic Paravertebral Block with Adjuvant Dexmedetomidine in Video-Assisted Thoracoscopic Surgery: A Randomized, Double-Blind Study
}

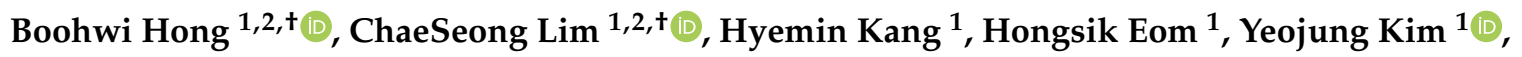 \\ Hyun Jin Cho ${ }^{3}{ }^{(1)}$, Woosik Han ${ }^{3}$, , Sunyeul Lee ${ }^{1,2}$, Woosuk Chung ${ }^{1,2}$ and Yoon-Hee Kim ${ }^{1,2, *}$ \\ 1 Department of Anesthesiology and Pain Medicine, Chungnam National University Hospital, 282 \\ Munhwa-ro, Jung-gu, Daejeon 35015, Korea; koho0127@gmail.com (B.H.); limtwo2@gmail.com (C.L.); \\ man4ok2017@naver.com (H.K.); tdoreins@naver.com (H.E.); yeojung80@naver.com (Y.K.); \\ neoquack@naver.com (S.L.); woosuk119@gmail.com (W.C.) \\ 2 Department of Anesthesiology and Pain Medicine, College of Medicine, Chungnam National University, \\ 266 Munhwa-ro, Jung-gu, Daejeon 35015, Korea \\ 3 Department of Thoracic \& Cardiovascular Surgery, Chungnam National University Hospital, \\ 282 Munhwa-ro, Jung-gu, Daejeon 35015, Korea; irainy79@naver.com (H.J.C.); innomi79@gmail.com (W.H.) \\ * Correspondence: yhkim040404@gmail.com; Tel.: +82-42-280-7840; Fax: +82-42-280-7968 \\ + These authors contributed equally to this work.
}

Received: 12 February 2019; Accepted: 7 March 2019; Published: 12 March 2019

\begin{abstract}
Background: The addition of the adjuvant dexmedetomidine to a nerve block improves the quality of the block and reduces perioperative opioid consumption. The aim of this study was to assess the effect of dexmedetomidine as an adjuvant for the thoracic paravertebral block (TPVB) in postoperative pain control after video-assisted thoracoscopic surgery (VATS). Methods: Sixty-six males, aged 15-40 years, with spontaneous pneumothorax scheduled for VATS wedge resection were enrolled. Following surgery, ultrasound-guided TPVB was performed on the T3 and T5 levels with $30 \mathrm{~mL}$ of $0.5 \%$ ropivacaine, plus adjuvant dexmedetomidine $50 \mu \mathrm{g}$ or normal saline. The primary outcome was cumulative fentanyl consumption at $24 \mathrm{~h}$. Pain severity, the requirement for additional rescue analgesics, hemodynamic variations, and side effects were also evaluated. Results: Median postoperative cumulative fentanyl consumption at $24 \mathrm{~h}$ was significantly lower in the dexmedetomidine group (122.6 (interquartile range (IQR) 94.5-268.0) $\mu \mathrm{g}$ vs. 348.1 (IQR, 192.8-459.2) $\mu \mathrm{g}, p$-value $=0.001)$ with a Hodges-Lehman median difference between groups of 86.2 (95\% confidence interval (CI), 4.2-156.4) $\mathrm{mg}$. Coughing numeric rating scale (NRS) was lower in the dexmedetomidine group at postoperative 2, 4, 8, and $24 \mathrm{~h}$. However, resting NRS differed significantly only after $4 \mathrm{~h}$ postoperative. Conclusions: Dexmedetomidine as an adjunct in TPVB provided effective pain relief and significantly reduced opioid requirement in VATS.
\end{abstract}

Keywords: dexmedetomidine; video-assisted thoracoscopic surgery (VATS); thoracic paravertebral block (TPVB); pain; postoperative

\section{Introduction}

Video-assisted thoracic surgery (VATS) has been widely used in the treatment of spontaneous pneumothorax. Minimally invasive surgery is more effective than open thoracotomy in reducing postoperative pain and complications, as well as shortening operation time and hospital stay [1]. However, the management of postoperative pain—especially early postoperative pain-remains a concern of many anesthesiologists and thoracic surgeons [2,3]. 
Thoracic paravertebral block (TPVB) may reduce postoperative opioid consumption and provide effective pain control [4-6]. The effect of TPVB on pain relief is similar to that of epidural analgesia, which is considered the gold standard [7,8], and the development of TPVB techniques using ultrasound has made it easier and more accessible [9]. However, the duration of the local anesthetic itself, such as ropivacaine, is limited to $8-14 \mathrm{~h}$ and the rebound pain after the nerve block wears off diminishes the analgesic benefit of the nerve block [10]. Dexmedetomidine, an $\alpha 2$ adrenergic receptor agonist developed as a short-term sedative [11], has shown sympatholytic, analgesic, and opioid-sparing effects, whilst adjuvant dexmedetomidine has been found to improve the duration and quality of nerve block [12-14].

It remains unclear whether TPVB reduces opioid consumption in VATS surgery $[2,5,15,16]$. Therefore, the present study investigated the effect of dexmedetomidine on the opioid-sparing properties of TPVB after VATS surgery.

\section{Materials and Methods}

The study protocol was approved by the Chungnam National University Hospital Institutional Review Board (IRB CNUH 2015-11-003-004), and the trial was registered at the Clinical Research Information Service, a clinical trial registry in Korea (KCT0001770). This parallel group, randomized, controlled, double-blind study enrolled patients scheduled for wedge resection under VATS at Chungnam National University Hospital (Daejeon, Korea), with all patients providing written informed consent.

All patients were males aged 15-40 years with American Society of Anesthesiologists physical status I or II scheduled for wedge resection under VATS. Patients were excluded if they refused TPVB, had a coagulopathy or bleeding disorder, were being treated with an antiplatelet agent, had a local infection at the injection site, were hypersensitive to local amide anesthetics, or were hypersensitive or allergic to dexmedetomidine. Patients were also excluded if they had central neuropathy, a body mass index $>35 \mathrm{~kg} / \mathrm{m}^{2}$, uncontrolled diabetes mellitus, significant cardiopulmonary disease, or psychiatric disease.

After induction of general anesthesia, the study subjects were randomized 1:1 to the dexmedetomidine ( $30 \mathrm{~mL}$ of $0.5 \%$ ropivacaine plus $0.5 \mathrm{~mL}$ of $50 \mu \mathrm{g}$ dexmedetomidine) group or control ( $30 \mathrm{~mL}$ of $0.5 \%$ ropivacaine plus $0.5 \mathrm{~mL}$ of normal saline) group using a computer-generated random number table with a block size of four. To conceal group allocation, the random number table was saved in Redcap software, which was used for randomization and data management. This randomization function in Redcap was accessible only to researchers preparing the study drugs; these researchers were not involved in patient monitoring or outcome analyses. The study drugs were prepared in a space other than the operating room in which surgery was performed.

On the day before surgery, a professional nurse inserted 18-gauge intravenous catheters into each patient's forearm, and the researcher explained the pain numeric rating scale (NRS) to the patient. Patients were premedicated with anticholinergics $(0.04 \mathrm{mg} / \mathrm{kg}$ of glycopyrrolate). Routine monitoring included electrocardiography, pulse oximetry, and noninvasive blood pressure measurements. Patients were anesthetized by standard methods using propofol, remifentanil, rocuronium, and sevoflurane. Patients were intubated with a 37 or 39 Fr double-lumen tube, with the optimal position of the tube and one-lung ventilation confirmed by bronchoscopy. Bronchoscopy was again performed after the patient was changed from the supine to the lateral decubitus position. One-lung ventilation was adjusted to $5-6 \mathrm{~mL} / \mathrm{kg}$ of ideal body weight, and the tidal volume and respiration rate were adjusted to $35-40 \mathrm{mmHg}$ of end-tidal $\mathrm{CO}_{2}$. Surgery was performed using either a single port or 2-3 ports, depending on the difficulty of surgery and the position of the lesion. At the end of the operation, a chest tube was inserted into the main port and lung expansion was confirmed directly by thoracoscopy, followed by a recruitment maneuver at $30 \mathrm{mmHg}$. 


\subsection{Thoracic Paravertebral Block}

During the completion of wound dressing immediately after surgery, the lateral decubitus position was maintained with two-lung ventilation under general anesthesia. All blocks were performed by a single researcher who was blinded to the group assignment and study drug. A 22 gauge, $80 \mathrm{~mm}$, echogenic needle (SonoPlex cannulas, Pajunk ${ }^{\circledR}$, Geisingen, Germany), MylabTM25 Gold (Esaote, Genova, Italy), and a linear probe (LA435: 6-18 MHz, Esaote, Genova, Italy) were used. Povidone-iodine was used to make an aseptic field. The locations of the spinous process, transverse process, and ribs were confirmed by ultrasound. The first rib was identified in the parasagittal plane, and the ribs were counted in order. The T3-4 intercostal space and the transverse process were confirmed by an in-plane intercostal approach as described by Yasuyuki Shibata [17]. The needle was located in the thoracic paravertebral space and $1 \mathrm{~mL}$ of test dose was applied to confirm the displacement of the pleura. The assistant subsequently injected $14 \mathrm{~mL}$ of prepared drug. The same procedure was applied to the fifth thoracic paravertebral space.

After two injections, the patient's position was changed to a supine position. The inhalation anesthetic was discontinued and muscle relaxation was reversed by pyridostigmine and glycopyrrolate. When the patient recovered consciousness and spontaneous breathing was sufficient, the endotracheal tube was removed. The level of mental status was monitored in the post-anesthesia care unit (PACU), taking into account the time that the drug had sufficiently spread. Successful TPVB was assessed around the level of the nipple by a pinprick test with a stylet of 22-gauge spinal needle $30 \mathrm{~min}$ after the block. During this assessment, the pain score was again explained to the patient and analgesics were administered through the patient-controlled analgesia (PCA) device (GemStar ${ }^{\mathrm{TM}}$, Hospira, IL, USA) when a patient reported an NRS score $\geq 4$. Patients who experienced bradycardia-defined as a heart rate $<50$ beats $/ \mathrm{min}$-were treated with $0.5 \mathrm{mg}$ of atropine.

To increase the reliability of the data, each PCA device was collected, and the log records stored in the device were transferred to an electronic medical records (EMRs) system. Each PCA device was set to administer a $0.5 \mu \mathrm{g} / \mathrm{kg}$ bolus dose of fentanyl without background infusion, with a lockout time of $15 \mathrm{~min}$ and a total allowable fentanyl dose of $1000 \mu \mathrm{g}$. If pain control was insufficient with PCA alone, patients were administered $30 \mathrm{mg}$ of ketorolac, followed, if necessary, by $25 \mathrm{mg}$ of pethidine. All patients received intravenous nefopam $20 \mathrm{mg}$ (12-h interval) as part of multimodal analgesia.

The primary outcome was postoperative cumulative fentanyl consumption at $24 \mathrm{~h}$. Secondary outcomes were the use of analgesics in the PACU, resting and coughing NRS over time, and maximum NRS during the $24 \mathrm{~h}$ study period.

\subsection{Statistical Analyses}

The required sample size was calculated based on a previous study [4,5] using G*Power (version 3.1, Franz Faul \& Edgar Erdfelder, Trier, Germany). In those studies, morphine consumption for $24 \mathrm{~h}$ after TPVB for VATS was approximately $30 \pm 10 \mathrm{mg}$, which was converted to the equivalent amount of fentanyl. A study using dexmedetomidine as an adjuvant in TPVB after breast cancer surgery found that the analgesic requirement was reduced by $25 \%$ [18]. Assuming a $25 \%$ reduction in fentanyl consumption and a power of $80 \%$ with a risk of 0.05 for type 1 errors (two-tailed, effect size of 0.75 ), the minimum number of patients required in each group was 29 . Allowing for a $10 \%$ dropout rate, the planned number of patients was 66.

All statistical analyses were performed using R software version 3.4.3 (R Project for Statistical Computing, Vienna, Austria). Normality was tested using the Shapiro-Wilk test. Continuous variables were analyzed by Student's $t$-test or the Mann-Whitney test, depending on the normality of the data, and recorded as mean \pm SD or median (interquartile range (IQR)). Point estimation and the confidence interval of the Hodges-Lehmann's median were calculated using SAS software (version 9.3 for Windows, SAS Korea, Seoul, Korea). Categorical variables were recorded as numbers (\%) and analyzed using the Chi-square test or Fisher's exact test. A two-tailed $p$-value of $<0.05$ was considered statistically significant. Repeated measurements were analyzed using repeated measures analysis of 
variance. If the differences were significant, Bonferroni's correction was used to reduce the probability of a type 1 error occurring when multiple testing was performed on each point.

\section{Results}

From January 2016 to December 2017, 98 patients were assessed for eligibility, and 32 were excluded. These 32 patients consisted of 11 patients who had surgery on both sides, 6 female patients, and 15 patients who refused study participation. The remaining 66 patients were randomized in a 1:1 ratio: 33 to the dexmedetomidine group and 33 to the control group. None of the participants were lost to follow-up. The Consolidated Standards of Reporting Trials (CONSORT) diagram is shown in Figure 1, followed by a comparison of the demographic and clinical characteristics of the two groups (Table 1).

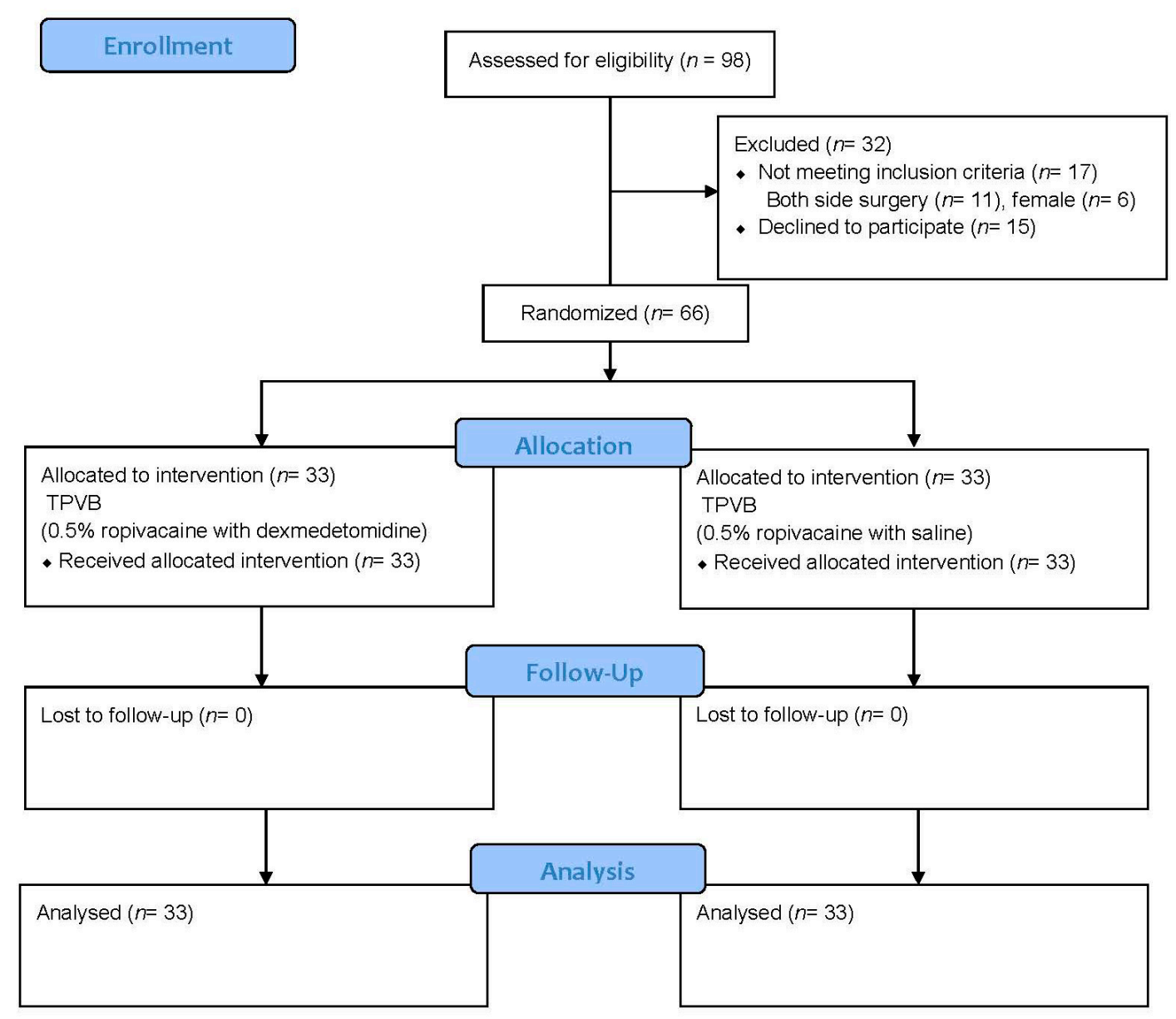

Figure 1. The Consolidated Standards of Reporting Trials (CONSORT) flow diagram of study participants. TPVB: thoracic paravertebral block. 
Table 1. Demographic and clinical characteristics of the study patients.

\begin{tabular}{ccc}
\hline & $\begin{array}{c}\text { Dexmedetomidine Group } \\
(\boldsymbol{n}=\mathbf{3 3})\end{array}$ & $\begin{array}{c}\text { Control Group } \\
(\boldsymbol{n}=\mathbf{3 3})\end{array}$ \\
\hline Age (year) & $19.0(17.0-22.0)$ & $19.0(18.0-22.0)$ \\
Height $(\mathrm{cm})$ & $173.3(169.5-178.2)$ & $173.1(166.6-178.2)$ \\
Weight $(\mathrm{kg})$ & $58.2 \pm 8.0$ & $57.8 \pm 8.1$ \\
BMI $\left(\mathrm{kg} / \mathrm{m}^{2}\right)$ & $19.2 \pm 2.2$ & $19.3 \pm 2.4$ \\
Port number & & \\
1 & $21(63.6)$ & $13(39.4)$ \\
2 & $2(6.1)$ & $4(12.1)$ \\
3 & $10(30.3)$ & $16(48.5)$ \\
Previous VATS history & $7(21.2)$ & $11(33.3)$ \\
Pleural adhesion & $3(9.1)$ & $9(27.3)$ \\
\hline
\end{tabular}

Variables described as median (interquartile range); mean \pm standard deviation (SD); or number (\%). BMI: body mass index; VATS: video-assisted thoracoscopic surgery.

Postoperative cumulative fentanyl consumption at $24 \mathrm{~h}$ was significantly lower in the dexmedetomidine group than in the control group (122.6 (IQR, 94.5-268.0) $\mu \mathrm{g}$ vs. 348.1 (IQR, 192.8-459.2) $\mu \mathrm{g}, p=0.001$ ), with a Hodges-Lehman median difference of 86.2 (95\% confidence interval (CI), 4.2-156.4) $\mathrm{mg}$ between groups. The number of patients requiring analgesics in the PACU was significantly lower in the dexmedetomidine group than in the control group $(12.1 \%$ vs. $42.4 \%, p=0.013$; relative risk $(95 \% \mathrm{CI})=0.368(0.15-0.899))$. The difference in opioid consumption, which began during the early postoperative period, continued over the entire $24 \mathrm{~h}$ postoperative period (Figure 2). NRS pain score for coughing was lower in the dexmedetomidine group at postoperative 2, 4, 8, and $24 \mathrm{~h}$. However, NRS pain score for resting was not significantly different at any point postoperatively, other than at $4 \mathrm{~h}$ (Figure 3). Maximum NRS pain scores for both resting and coughing over $24 \mathrm{~h}$ were significantly lower in the dexmedetomidine group (Table 2).

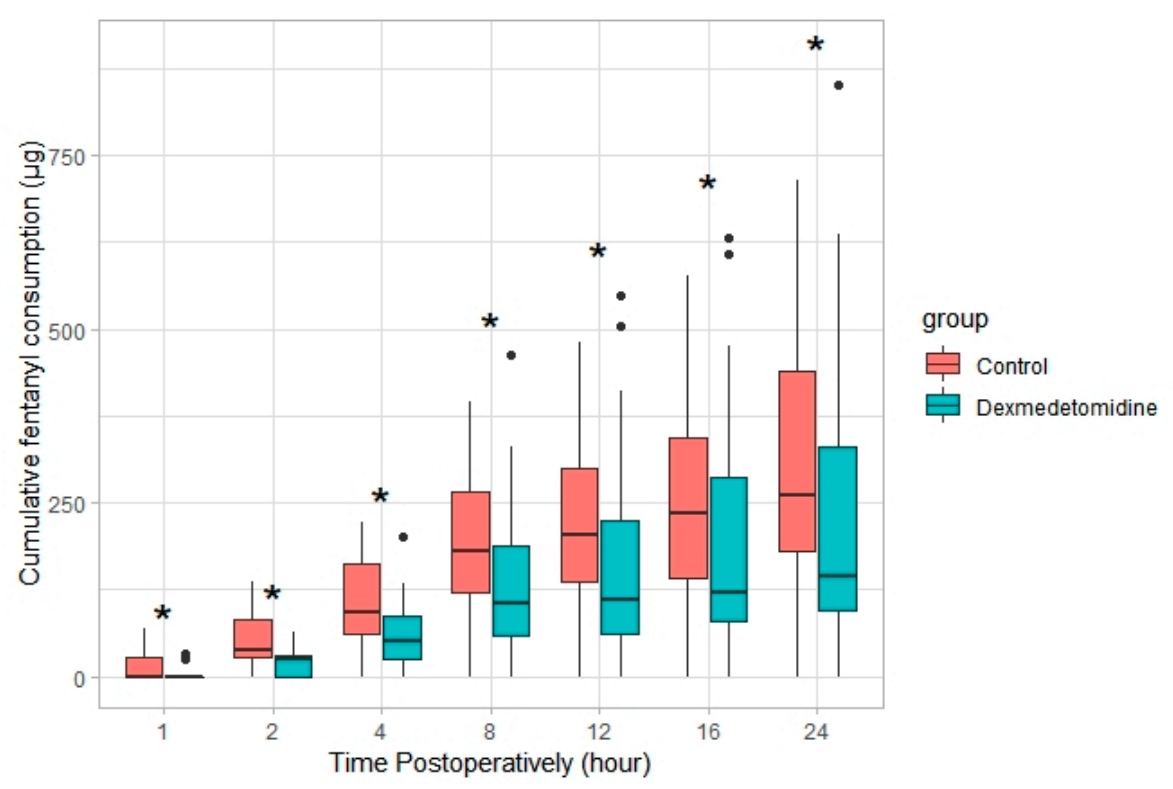

Figure 2. Cumulative fentanyl consumption over time in both groups. Data are expressed as median (interquartile range). ${ }^{*} p<0.05$. $\bullet$ outlier (any data point more than 1.5 interquartile ranges below the first quartile or above the third quartile). 


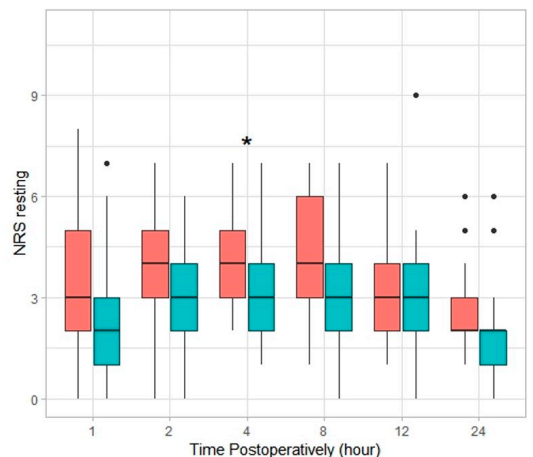

(a)

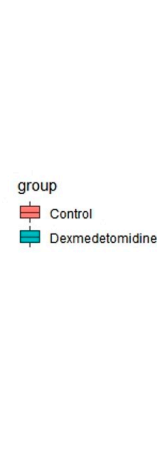

(b)

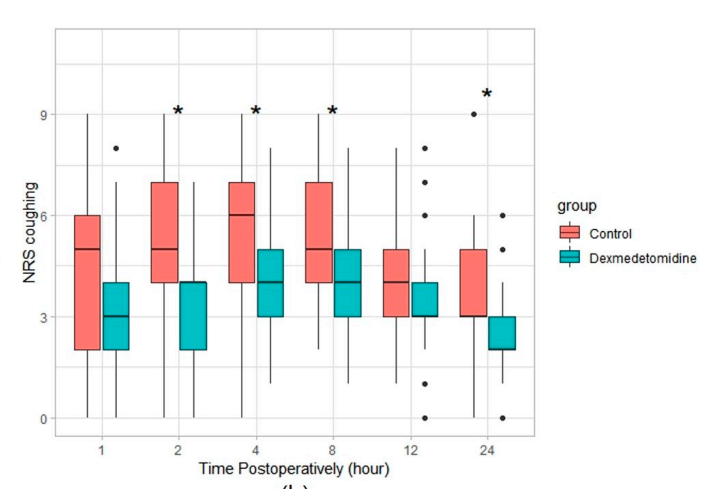

(b)

Figure 3. Pain score over time while (a) resting and (b) coughing. Data are expressed as median (interquartile range). ${ }^{*} p<0.05$. $\bullet$ outlier (any data point more than 1.5 interquartile ranges below the first quartile or above the third quartile).

Table 2. Postoperative results in the study patients.

\begin{tabular}{cccc}
\hline & Dexmedetomidine Group $(\boldsymbol{n}=\mathbf{3 3})$ & Control Group $(\boldsymbol{n}=\mathbf{3 3})$ & $p$ \\
\hline Extubation time (min) & $11.0(9.0-13.0)$ & $10.0(8.0-12.0)$ & 0.071 \\
PACU stay time (min) & $44.0(39.0-53.0)$ & $42.0(38.0-49.0)$ & 0.517 \\
Analgesic request in PACU & $4(12.1 \%)$ & $14(42.4 \%)$ & 0.013 \\
Maximum NRS pain score & & & \\
Resting & $4.0(3.0-5.0)$ & $5.0(4.0-6.0)$ & 0.032 \\
Coughing & $5.0(4.0-6.0)$ & $7.0(5.0-7.0)$ & 0.003 \\
Rescue analgesic (ketorolac) & $8(24.2 \%)$ & $7(21.2 \%)$ & 1.000 \\
Rescue analgesic (pethidine) & $1(3.0 \%)$ & $3(9.1 \%)$ & 0.606 \\
Discharge day after operation & $3.0(3.0-5.0)$ & $3.0(3.0-5.0)$ & 0.455 \\
Nausea & $4(12.1 \%)$ & $3(9.1 \%)$ & 1.000
\end{tabular}

Data are presented as median (interquartile range) or number (\%). NRS: numeric rating scale; PACU: post-anesthesia care unit.

The frequency of additional rescue analgesics, length of hospital stay, and postoperative nausea were not different between the two groups (Table 2). Although postoperative heart rate was lower in the dexmedetomidine group, no patient required atropine (Figure 4).

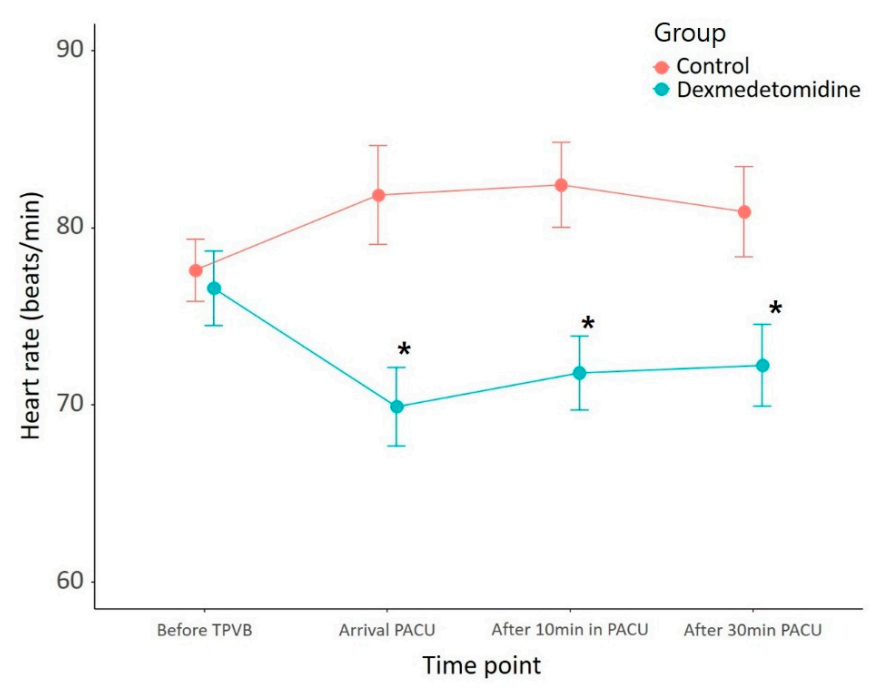

Figure 4. Changes in heart rate over time in the post-anesthesia care unit. The changes over time differed significantly in the two groups $(p=0.002) .{ }^{*} p<0.013$ using Bonferroni's correction for multiple comparisons. PACU: post-anesthesia care unit; TPVB: thoracic paravertebral block. 


\section{Discussion}

This study found that dexmedetomidine as an adjuvant for TPVB for postoperative pain control after VATS had superior analgesic efficacy compared to TPVB alone. Effective pain control during the acute phase reduced the use of analgesics for up to $24 \mathrm{~h}$.

The mechanism of adjuvant dexmedetomidine for nerve block is not fully understood, but it is likely to be multifactorial. The lower pain score in the early postoperative period and reduced opioid consumption up to $24 \mathrm{~h}$ postoperatively are thought to be a mixed effect of several mechanisms. Dexmedetomidine has centrally mediated analgesic effects at the cerebral and spinal levels through an $\alpha 2$-receptor mechanism. Its direct binding to $\alpha 2$ adrenoreceptors in the locus coeruleus may explain the extended effects of intravenous dexmedetomidine on nerve block duration [19]. A trial in patients undergoing arthroscopic shoulder surgery showed that systemic dexmedetomidine prolonged interscalene block compared to perineural dexmedetomidine [13]. The peripheral effects of $\alpha 2$ agonists resulting in analgesia are mediated by a reduction in the release of norepinephrine as well as by $\alpha 2$ receptor-independent inhibition of nerve fiber action potentials. According to Fritsch et al. [20], the perineurally injected dexmedetomidine prolonged nerve block for several hours, but blood concentration of dexmedetomidine was very low, so a systemic mechanism is not considered to block prolongation. Also, the bilateral paired study of controlling for systemic effects found that the duration of saphenous nerve block was significantly longer in the adjuvant dexmedetomidine plus ropivacaine [21]. Perineural dexmedetomidine prolonged sensory block of the ulnar nerve by $60 \%$, and systemic dexmedetomidine also prolonged sensory block by 10\% compared with placebo [22].

Because this study was a parallel group study with ropivacaine alone as the active comparison group, dexmedetomidine was not intravenously injected into patients. Therefore, it is difficult to determine the precise mechanism by which adjuvant dexmedetomidine has opioid-sparing effects in TPVB. Dexmedetomidine has an intrinsic analgesic effect. Therefore, in the absence of a study group administered dexmedetomidine alone (without ropivacaine) or sham block (placebo control), it was not possible to determine whether the observed benefits were due to the intrinsic systemic analgesic effect of dexmedetomidine or to its potentiation of the analgesic effect of the nerve block.

TPVB has been shown to reduce various complications of the epidural block in patients undergoing breast surgery, and is considered the gold standard [23]. However, it is unclear whether opioid consumption is reduced after TPVB in breast surgery, with studies showing that its effect is limited to the early postoperative period, suggesting that it is due to the duration of ropivacaine anesthesia [24,25]. The adjuvant dexmedetomidine, which increases the quality and duration of TPVB, has been consistently reported to reduce postoperative opioid consumption in breast surgery [18,26]. In VATS, it is also not clear whether TPVB reduces opioid consumption. A single TPVB was reported effective in controlling pain for up to $6 \mathrm{~h}$ [2], but there was no difference in morphine consumption after $6 \mathrm{~h}$. In addition, a single TPVB did not reduce opioid consumption at 24 and $48 \mathrm{~h}$ after VATS surgery [15]. Preoperative multiple-injection TPVB, however, significantly reduced cumulative morphine consumption for $48 \mathrm{~h}$ postoperatively [5]. The reason for these conflicting results may have been due to differences in study design, differences in the number of injections, the volume of local anesthetics administered, the number of ports, the patient population, and operation times. Furthermore, nociceptive pathways in thoracic surgery are complex [27]. Nociceptive somatic afferents are conveyed by intercostal nerves after skin incision, rib retraction, muscle splitting, injury to the parietal pleura, and chest drain insertion into the ipsilateral dorsal horn of the spinal cord (T4-T10). Pain signals of the diaphragmatic pleura are transmitted by the phrenic nerve, usually expressed as ipsilateral shoulder pain. Additionally, insertion of the tube can irritate the pleural dome, causing postoperative pain. The vagus nerve running along the pericardium also controls pain of mediastinal pleural origin. As pain after thoracic surgery is caused by multiple pathways, TPVB alone is insufficient for complete pain control, which may explain the differences in results observed in previous studies. 
This study has several limitations. Firstly, all patients in the dexmedetomidine group received $50 \mu \mathrm{g}$ of dexmedetomidine. As $200 \mu \mathrm{g}$ of dexmedetomidine was dissolved in $2 \mathrm{~mL}$ saline, it was difficult to fine-tune the dose. Instead, using a small volume of dexmedetomidine could have minimal effect on the concentration of local anesthetics. Most studies have used weight-based dosing of dexmedetomidine $(1 \mu \mathrm{g} / \mathrm{kg})$, and similar doses were applied to the lean populations in this study. Another limitation was the lack of a dose-response study, which likely would have shown that higher doses are more effective. However, a previous study found that a high intravenous dose of $2 \mu \mathrm{g} / \mathrm{kg}$ was associated with a greater need of ephedrine for intraoperative hypotension, suggesting a trade-off between the benefits and risks of high-dose adjuvant dexmedetomidine [28]. This study also did not evaluate the dermatomal spread of the blockade representing its analgesic quality. Analgesia and the spread of local anesthetic can be inconsistent. In addition, satisfactory postoperative analgesia may be achieved after the block, although targeted dermatomes are only partially blocked [29]. However, this study tried to eliminate the sparing level by administering a large volume of $15 \mathrm{~mL}$ to the third and fifth levels. Another limitation of this study was its inclusion of male patients only. Females have been reported to feel more severe, frequent, and diffuse pain than males with similar disease processes [30]. The differences in pain sensitivity, pain facilitation, and pain inhibition may be due to sex hormones, endogenous opioid function, genetic factors, psychosocial processes such as pain coping and catastrophizing, and gender roles [31]. Also, pneumothorax is much more prevalent in males [32]. By assessing males only, this study was able to reduce any bias resulting from sex differences in pain.

\section{Conclusions}

Dexmedetomidine as an adjunct in TPVB provided effective pain relief and significantly reduced opioid requirement in VATS.

Author Contributions: Conceptualization, B.H., C.L., H.K, Y.-H.K.; methodology, B.H. and Y.K.; software, S.L.; validation, H.J.C., W.H.; formal analysis, B.H. W.C.; investigation, Y.-H.K.; resources, H.E.; data curation, H.K., H.E.; writing-original draft preparation, B.H.; writing-review and editing, C.L., W.C., Y.-H.K.; visualization, B.H.; supervision, Y.-H.K.; project administration, B.H.; funding acquisition, B.H.

Funding: This research was partially supported by Chungnam National University Hospital Research Fund, 2018-CF-024.

Acknowledgments: The authors thank, In-Sun Kwon (Clinical Trials Center, Chungnam National University Hospital) and AdjunctSangil Park (Department of Anesthesiology and Pain Medicine, College of Medicine, Chungnam National University) for their invaluable advice throughout this study.

Conflicts of Interest: The authors declare no conflict of interest.

\section{References}

1. Grogan, E.L.; Jones, D.R. VATS lobectomy is better than open thoracotomy: What is the evidence for short-term outcomes? Thorac. Surg. Clin. 2008, 18, 249-258. [CrossRef] [PubMed]

2. Hill, S.E.; Keller, R.A.; Stafford-Smith, M.; Grichnik, K.; White, W.D.; D'Amico, T.A.; Newman, M.F. Efficacy of single-dose, multilevel paravertebral nerve blockade for analgesia after thoracoscopic procedures. Anesthesiology 2006, 104, 1047-1053. [CrossRef] [PubMed]

3. Kotemane, N.C.; Gopinath, N.; Vaja, R. Analgesic techniques following thoracic surgery: A survey of United Kingdom practice. Eur. J. Anaesthesiol. 2010, 27, 897-899. [CrossRef] [PubMed]

4. Kaya, F.N.; Turker, G.; Mogol, E.B.; Bayraktar, S. Thoracic paravertebral block for video-assisted thoracoscopic surgery: Single injection versus multiple injections. J. Cardiothorac. Vasc. Anesth. 2012, 26, 90-94. [CrossRef] [PubMed]

5. Kaya, F.N.; Turker, G.; Basagan-Mogol, E.; Goren, S.; Bayram, S.; Gebitekin, C. Preoperative multiple-injection thoracic paravertebral blocks reduce postoperative pain and analgesic requirements after video-assisted thoracic surgery. J. Cardiothorac. Vasc. Anesth. 2006, 20, 639-643. [CrossRef] [PubMed] 
6. Thavaneswaran, P.; Rudkin, G.E.; Cooter, R.D.; Moyes, D.G.; Perera, C.L.; Maddern, G.J. Brief reports: Paravertebral block for anesthesia: A systematic review. Anesth. Analg. 2010, 110, 1740-1744. [CrossRef] [PubMed]

7. Pintaric, T.S.; Potocnik, I.; Hadzic, A.; Stupnik, T.; Pintaric, M.; Novak Jankovic, V. Comparison of continuous thoracic epidural with paravertebral block on perioperative analgesia and hemodynamic stability in patients having open lung surgery. Reg. Anesth. Pain Med. 2011, 36, 256-260. [CrossRef] [PubMed]

8. Baidya, D.K.; Khanna, P.; Maitra, S. Analgesic efficacy and safety of thoracic paravertebral and epidural analgesia for thoracic surgery: A systematic review and meta-analysis. Interact. Cardiovasc. Thorac. Surg. 2014, 18, 626-635. [CrossRef] [PubMed]

9. SC, O.R.; Donnell, B.O.; Cuffe, T.; Harmon, D.C.; Fraher, J.P.; Shorten, G. Thoracic paravertebral block using real-time ultrasound guidance. Anesth. Analg. 2010, 110, 248-251. [CrossRef]

10. Williams, B.A.; Bottegal, M.T.; Kentor, M.L.; Irrgang, J.J.; Williams, J.P. Rebound pain scores as a function of femoral nerve block duration after anterior cruciate ligament reconstruction: Retrospective analysis of a prospective, randomized clinical trial. Reg. Anesth. Pain Med. 2007, 32, 186-192. [CrossRef] [PubMed]

11. Gerlach, A.T.; Dasta, J.F. Dexmedetomidine: An updated review. Ann. Pharmacother. 2007, 41, $245-252$. [CrossRef]

12. Abdallah, F.W.; Brull, R. Facilitatory effects of perineural dexmedetomidine on neuraxial and peripheral nerve block: A systematic review and meta-analysis. Br. J. Anaesth. 2013, 110, 915-925. [CrossRef] [PubMed]

13. Abdallah, F.W.; Dwyer, T.; Chan, V.W.; Niazi, A.U.; Ogilvie-Harris, D.J.; Oldfield, S.; Patel, R.; Oh, J.; Brull, R. IV and Perineural Dexmedetomidine Similarly Prolong the Duration of Analgesia after Interscalene Brachial Plexus Block: A Randomized, Three-arm, Triple-masked, Placebo-controlled Trial. Anesthesiology 2016, 124, 683-695. [CrossRef]

14. Bae, H.B. Dexmedetomidine: An attractive adjunct to anesthesia. Korean J. Anesthesiol. 2017, 70, 375-376. [CrossRef]

15. Vogt, A.; Stieger, D.S.; Theurillat, C.; Curatolo, M. Single-injection thoracic paravertebral block for postoperative pain treatment after thoracoscopic surgery. Br. J. Anaesth. 2005, 95, 816-821. [CrossRef]

16. Xu, J.; Yang, X.; Hu, X.; Chen, X.; Zhang, J.; Wang, Y. Multilevel Thoracic Paravertebral Block Using Ropivacaine with/without Dexmedetomidine in Video-Assisted Thoracoscopic Surgery. J. Cardiothorac. Vasc. Anesth. 2018, 32, 318-324. [CrossRef]

17. Shibata, Y.; Nishiwaki, K. Ultrasound-guided intercostal approach to thoracic paravertebral block. Anesth. Analg. 2009, 109, 996-997. [CrossRef]

18. Mohamed, S.A.; Fares, K.M.; Mohamed, A.A.; Alieldin, N.H. Dexmedetomidine as an adjunctive analgesic with bupivacaine in paravertebral analgesia for breast cancer surgery. Pain Physician 2014, 17, E589-E598.

19. Guo, T.Z.; Jiang, J.Y.; Buttermann, A.E.; Maze, M. Dexmedetomidine injection into the locus ceruleus produces antinociception. Anesthesiology 1996, 84, 873-881. [CrossRef]

20. Fritsch, G.; Danninger, T.; Allerberger, K.; Tsodikov, A.; Felder, T.K.; Kapeller, M.; Gerner, P.; Brummett, C.M. Dexmedetomidine added to ropivacaine extends the duration of interscalene brachial plexus blocks for elective shoulder surgery when compared with ropivacaine alone: A single-center, prospective, triple-blind, randomized controlled trial. Reg. Anesth. Pain Med. 2014, 39, 37-47. [CrossRef]

21. Andersen, J.H.; Grevstad, U.; Siegel, H.; Dahl, J.B.; Mathiesen, O.; Jaeger, P. Does Dexmedetomidine Have a Perineural Mechanism of Action When Used as an Adjuvant to Ropivacaine?: A Paired, Blinded, Randomized Trial in Healthy Volunteers. Anesthesiology 2017, 126, 66-73. [CrossRef]

22. Marhofer, D.; Kettner, S.C.; Marhofer, P.; Pils, S.; Weber, M.; Zeitlinger, M. Dexmedetomidine as an adjuvant to ropivacaine prolongs peripheral nerve block: A volunteer study. Br. J. Anaesth. 2013, 110, 438-442. [CrossRef]

23. Woodworth, G.E.; Ivie, R.M.J.; Nelson, S.M.; Walker, C.M.; Maniker, R.B. Perioperative Breast Analgesia: A Qualitative Review of Anatomy and Regional Techniques. Reg. Anesth. Pain Med. 2017, 42, 609-631. [CrossRef]

24. Shimizu, H.; Kamiya, Y.; Nishimaki, H.; Denda, S.; Baba, H. Thoracic paravertebral block reduced the incidence of chronic postoperative pain for more than 1 year after breast cancer surgery. JA Clin. Rep. 2015, 1, 19. [CrossRef]

25. Boughey, J.C.; Goravanchi, F.; Parris, R.N.; Kee, S.S.; Kowalski, A.M.; Frenzel, J.C.; Bedrosian, I.; Meric-Bernstam, F.; Hunt, K.K.; Ames, F.C.; et al. Prospective randomized trial of paravertebral block for patients undergoing breast cancer surgery. Am. J. Surg. 2009, 198, 720-725. [CrossRef] 
26. Mohta, M.; Kalra, B.; Sethi, A.K.; Kaur, N. Efficacy of dexmedetomidine as an adjuvant in paravertebral block in breast cancer surgery. J. Anesth. 2016, 30, 252-260. [CrossRef]

27. Daly, D.J.; Myles, P.S. Update on the role of paravertebral blocks for thoracic surgery: Are they worth it? Curr. Opin. Anaesthesiol. 2009, 22, 38-43. [CrossRef]

28. Kang, R.; Jeong, J.S.; Yoo, J.C.; Lee, J.H.; Choi, S.J.; Gwak, M.S.; Hahm, T.S.; Huh, J.; Ko, J.S. Effective Dose of Intravenous Dexmedetomidine to Prolong the Analgesic Duration of Interscalene Brachial Plexus Block: A Single-Center, Prospective, Double-Blind, Randomized Controlled Trial. Reg. Anesth. Pain Med. 2018. [CrossRef]

29. Naja, Z.M.; El-Rajab, M.; Al-Tannir, M.A.; Ziade, F.M.; Tayara, K.; Younes, F.; Lonnqvist, P.A. Thoracic paravertebral block: Influence of the number of injections. Reg. Anesth. Pain Med. 2006, 31, 196-201. [CrossRef]

30. Hurley, R.W.; Adams, M.C. Sex, gender, and pain: An overview of a complex field. Anesth. Analg. 2008, 107, 309-317. [CrossRef]

31. Bartley, E.J.; Fillingim, R.B. Sex differences in pain: A brief review of clinical and experimental findings. Br. J. Anaesth. 2013, 111, 52-58. [CrossRef]

32. Bobbio, A.; Dechartres, A.; Bouam, S.; Damotte, D.; Rabbat, A.; Regnard, J.F.; Roche, N.; Alifano, M. Epidemiology of spontaneous pneumothorax: Gender-related differences. Thorax 2015, 70, 653-658. [CrossRef]

(C) 2019 by the authors. Licensee MDPI, Basel, Switzerland. This article is an open access article distributed under the terms and conditions of the Creative Commons Attribution (CC BY) license (http:/ / creativecommons.org/licenses/by/4.0/). 